\title{
Incidence of arteriosclerosis in patients with rheumatoid arthritis receiving long-term corticosteroid therapy
}

\author{
K. KALBAK \\ King Christian X Hospital for Rheumatic Diseases, Graasten, Denmark
}

It is well known that long-term treatment with corticosteroids may have undesired side-effects. This paper reports an observation which, in all probability, should be interpreted as one more late side-effect that may be expected to occur in the course of prolonged treatment with these hormones.

In a great number of patients with rheumatoid arthritis, receiving this long-term treatment, ${ }^{*}$ routine $x$ ray examination of the ankle joint region revealed extensive calcification of the anterior and posterior tibial arteries and their ramifications.

The present investigation was undertaken in order to establish whether these arterial changes occurred more frequently and were more widespread in steroid-treated patients than in a control group consisting of patients who were not treated with corticosteroids.

\section{Materials and methods}

The study included a total of 334 patients, divided in two main groups (Table I):

\section{GROUP 1 (steroid-treated)}

86 patients with rheumatoid arthritis.

All receiving long-term treatment with a corticosteroid.

G R OU P 2 (control-group, not steroid-treated)

248 patients:

A: 138 patients with rheumatoid arthritis.

B: 110 patients suffering from various rheumatic diseases other than rheumatoid arthritis.

\section{Occurrence of arteriosclerosis}

The arteriosclerosis is observed particularly clearly in the lateral projection of the ankle joint.

By adopting a softer exposure technique it was possible to observe various degrees of arteriosclerosis, ranging from the merest trace to a massive and almost unbroken linear picture of the complete arterial plexus in the ankle joint region (Fig. 1, opposite).

In this manner arteriosclerosis was demonstrated in about 60 per cent. of the steroid-treated patients in Group 1, but only in about 20 per cent. among the control patients in Groups 2A and 2B (see Table II).

Already at an early stage of the investigation it became obvious that arteriosclerosis occurred not only more frequently, but also more extensively in the steroid-treated patients.

By standardizing the examination (films of the same size, placing the ankle joint upon the center of the film casette and using the same soft exposure), the different examinations could be compared. It was then possible to measure directly the linear extent of the arteriosclerosis. The results were then graduated in seven different grades, each representing an increase of $100 \mathrm{~mm}$. of arteriosclerosis in linear length (Fig. 2 and Table III, overleaf).

In the control groups more than 90 per cent. of the arteriosclerosis occurs within grades 1 and 2 , i.e. the extent of sclerotic arteries (the sum of the extent

Table I Distribution of 334 patients by age and sex

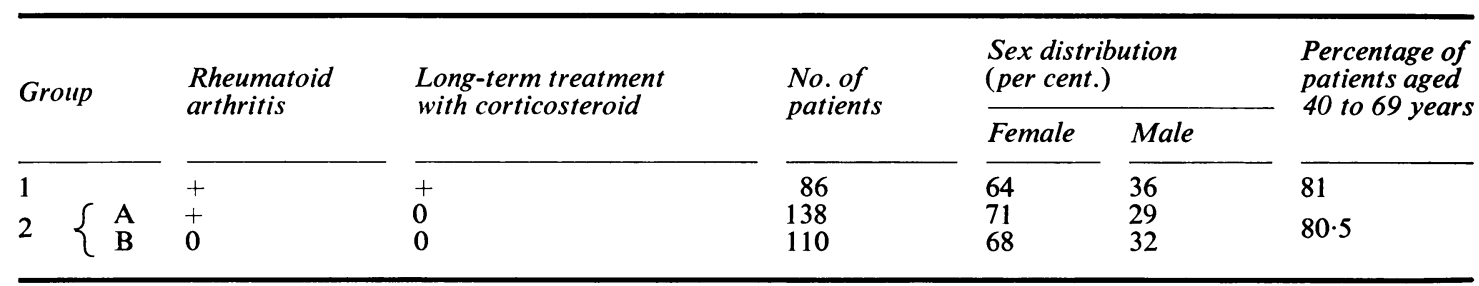

* Long-term treatment here means continuous administration of a corticosteroid for a period exceeding 6 months. 


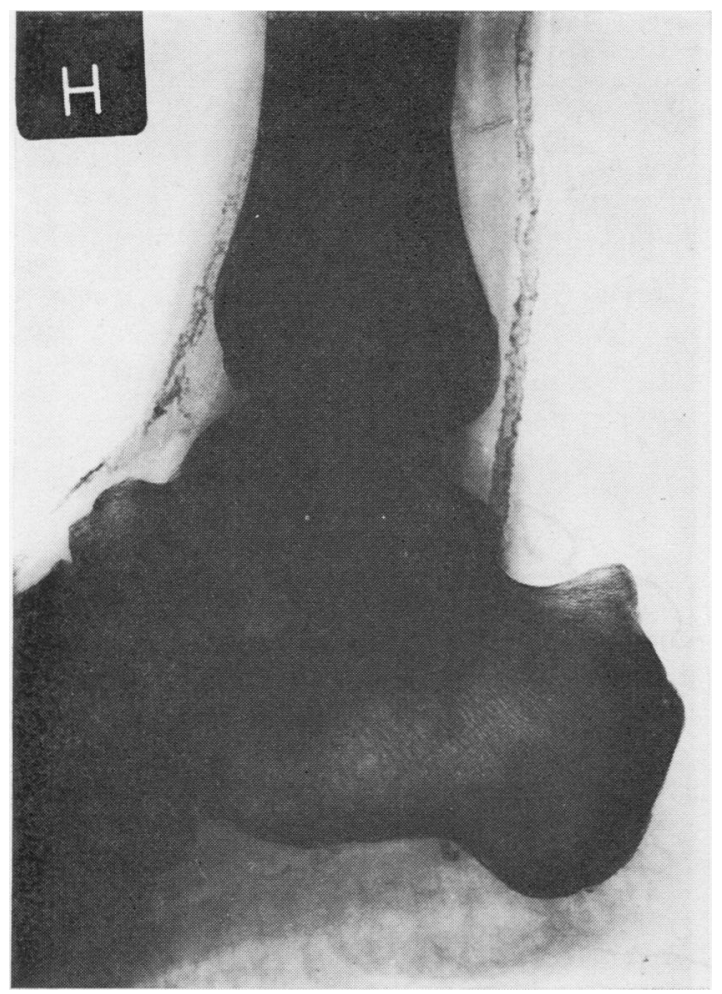

FIG. 1 Arteriosclerosis in the ankle joint region in a man aged 66 years who had been treated with corticosteroids for 12 years

measured in the right and in the left ankle region) is here relatively small (max. $200 \mathrm{~mm}$.).

In Group 1, however, only about half the cases of arteriosclerosis came into grades 1 and 2 , and the other cases were much more severely affected (Grades 3 to 7 ).

No definite relationship between the incidence and the extent of arteriosclerosis (in grades), the duration of the steroid treatment, and the ages of the patients was observed. The more extensive degrees of arterioscleriosis did not occur chiefly among the oldest patients, or in those who has been treated with steroids for the greatest length of time. In some cases severe arteriosclerosis could be seen after a relatively short

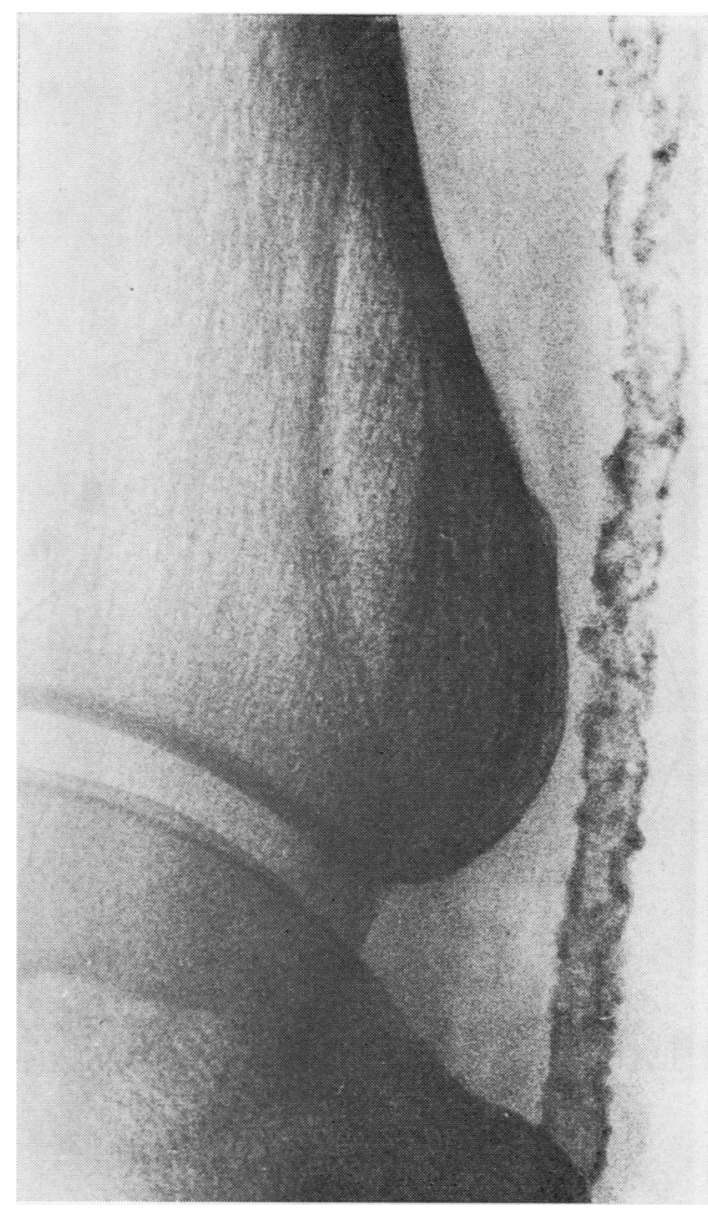

period of treatment, and in others who had received corticosteroids for more than 10 years the arteriosclerosis was of only minor degree.

It should also be emphasized, that there exist patients who have received steroids for up to 17 years without the slightest trace of arteriosclerosis being demonstrable.

For identifying the type of the arteriosclerosis, a biopsy was taken from the posterior tibial artery of a 60-year-old man who had suffered from a classical rheumatoid arthritis for 16 years, and had received

Table II Occurrence of arteriosclerosis

\begin{tabular}{|c|c|c|c|c|c|c|c|}
\hline \multirow{3}{*}{ Group } & & \multicolumn{3}{|l|}{ All ages (334) } & \multicolumn{3}{|c|}{ Age group 40 to 69 years $(280)$} \\
\hline & & \multirow[t]{2}{*}{ No. of patients } & \multicolumn{2}{|c|}{ Arteriosclerosis } & \multirow[t]{2}{*}{ No. of patients } & \multicolumn{2}{|c|}{ Arteriosclerosis } \\
\hline & & & No. & Per cent. & & No. & Per cent. \\
\hline $\begin{array}{l}1 \\
2\end{array}$ & $\begin{array}{l}\mathbf{A} \\
\mathbf{B}\end{array}$ & $\begin{array}{r}86 \\
138 \\
110\end{array}$ & $\begin{array}{l}53 \\
25 \\
23\end{array}$ & $\left.\begin{array}{l}62 \\
18 \\
21\end{array}\right\} 19 \cdot 5$ & $\begin{array}{l}78 \\
117 \\
85\end{array}$ & $\begin{array}{l}50 \\
23 \\
19\end{array}$ & $\left.\begin{array}{l}64 \\
19 \\
22\end{array}\right\} 20 \cdot 5$ \\
\hline
\end{tabular}



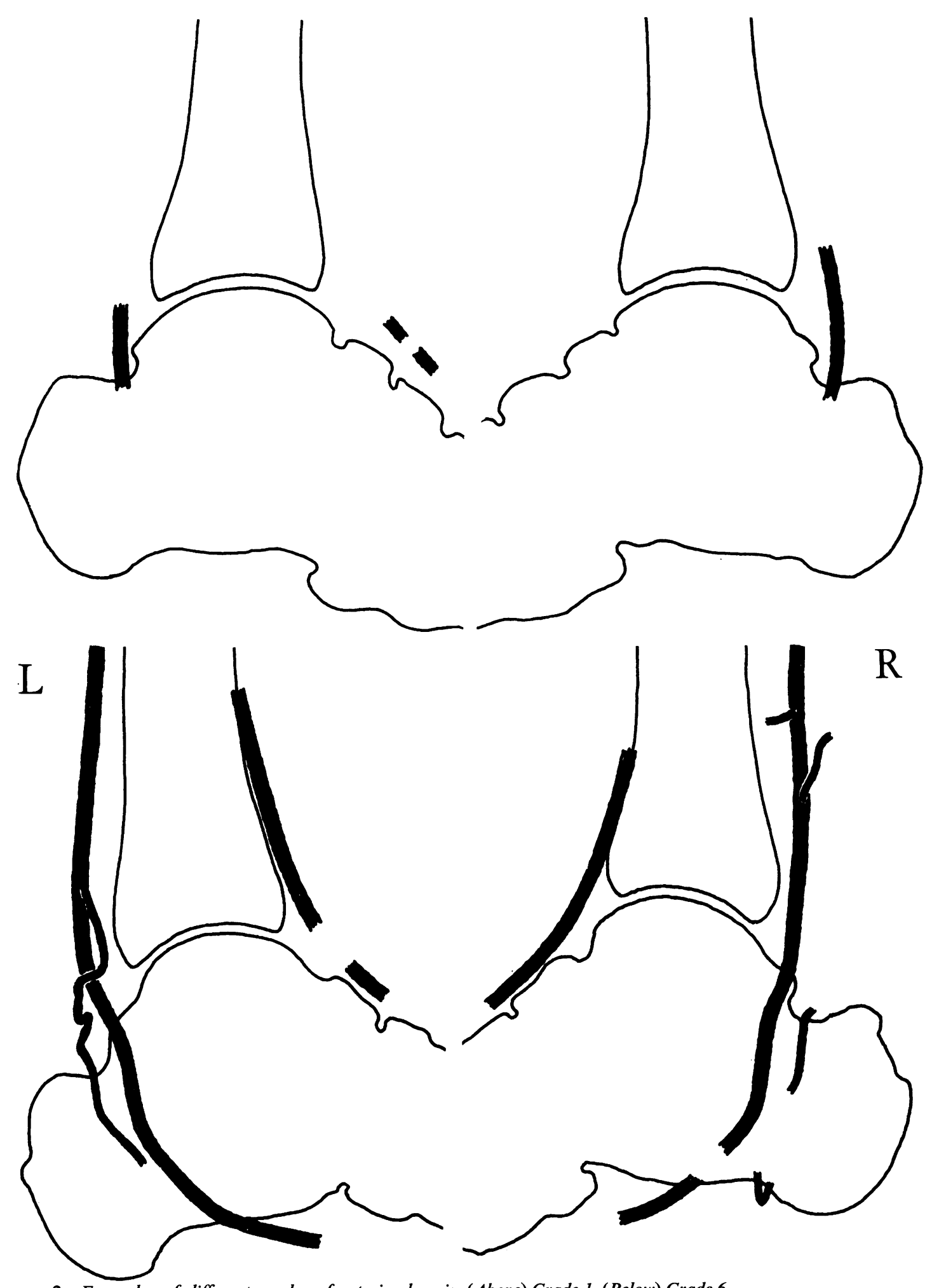

FIG. 2 Examples of different grades of arteriosclerosis. (Above) Grade 1. (Below) Grade 6 
Table III Grades of arteriosclerosis

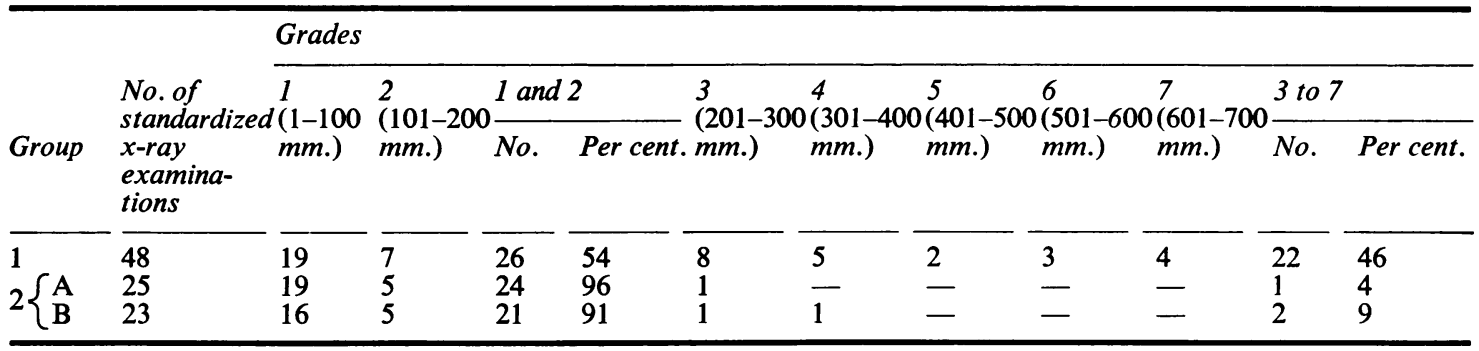

long-term treatment with corticosteroids for the last 12 years. The arteriosclerosis observed by $x$ ray examination was Grade 6.

The histological report (Dr. G. Teilum) was as follows:

'Biopsy from posterior tibial artery. Length: $20 \mathrm{~mm}$. diameter: $4 \mathrm{~mm}$. The lumen is measured at about $0.5 \mathrm{~mm}$. and the vessel has been transformed into a stiff calcareous tube.

The decalcified arterial tissue shows severe changes. Especially in the media there are numerous calcium deposits which are stained blue with haematoxylin and eosin. The deposits are in part confluent, and are surrounded by more irregular, minor aggregates. The lesions are not located to the media alone, as there is also a marked fibrous thickening of the intima, contributing to the narrowing of the lumen. Thus protruding fibrous intimal pads are seen sporadically luminal to the lamina elastica interna. No thrombus formation. No inflammatory lesions. The vessels of tunica adventitia show no changes. In one single spot lipoid-containing cell clusters were found in the intima and in the adventitia. Amyloid stainings with Congo red and methyl violet were both negative.

DIAG Nosis Arterial biopsy from the posterior tibial artery with excessive calcium deposits in the media, and fibrous proliferation of the intima.'

It is fairly reasonable to assume that the other cases of arteriosclerosis disclosed in the present study will exhibit changes similar to those described above.

\section{Results}

(a) Radiologically visible arteriosclerosis in the ankle joint region is three times as frequent in patients with rheumatoid arthritis receiving long-term treatment with corticosteroid as in controls.

(b) Furthermore, the arteriosclerosis is considerably more widespread in the steroid-treated patients than in the controls.

(c) A direct relationship between the extent of arteriosclerosis and the duration of steroid therapy, or the age of the patient, cannot be observed.

(d) Most of the calcium deposits are located in the media of the arteries. In comparison, the intimal changes are relatively insignificant, and lipoidcontaining deposits seem to occur only here and there and to a very small extent.

\section{Discussion}

To the best of my knowledge there are no publications dealing specifically with the relationship between long-term corticosteroid therapy and arteriosclerosis in patients with rheumatoid arthritis.

Adlersberg, Schaefer, and Drachman (1950) demonstrated a pathological increase in the serum cholesterol in 77 per cent. of 47 patients who were treated with either cortisone or ACTH. They thought that this might lead to early arteriosclerosis in patients receiving long-term treatment with these drugs. Unfortunately, the number of patients was relatively small, and the majority of them suffered from collagen disorders (systemic lupus erythematosus, polyarteritis, scleroderma, dermatomyositis), and only a few from rheumatoid arthritis.

Although Gardner (1965), in his comprehensive monograph, recognized the occurrence of pathological arterial changes in patients with rheumatoid arthritis, he maintained that it was hardly a matter of specific changes developing in this disease only. He thought that arteriosclerosis was as frequent and widespread in other diseases affecting the same age groups as in rheumatoid arthritis.

A communication from Finland (Skrifvars, Laine, and Wegelius, 1969) reported the incidence of arteriosclerosis of the extremities in patients with rheumatoid arthritis to be 15.4 per cent. compared to 3.4 per cent. in normal subjects. Unfortunately, no special mention was made of its incidence in the arthritic patients who had received long-term treatment with corticosteroids. They believed that the calcium deposits in the arterial wall were preceded by degenerative changes in the connective tissue which were probably part of the general mesenchymal disorder.

Judging from the results here presented, it is beyond doubt that arteriosclerosis is much more frequent, and considerably more widespread, in the steroid-treated patients with rheumatoid arthritis (Group 1), than in the controls (Group 2A and B). 
That steroid therapy as such is the causative factor is evident from the fact that the incidence of arteriosclerosis in those patients who also suffered from rheumatoid arthritis but were not treated with steroids (Group 2A) corresponds closely to that in the normal subjects (Group 2B).

Rheumatoid arthritis does not seem to affect the development of these characteristic arterial calcifications, and we are justified in regarding the pathological arterial lesions as one more side-effect of long-term treatment with corticosteroids. In the present study the incidence of this side-effect was about 60 per cent.

It was surprising that there seemed to be no overt clinical vascular symptoms despite the severe and widespread arterial lesions revealed by the radiographs.

On the face of it, one might expect to see typical evidence of arterial occlusion, but this was not the case. None of our patients complained of circulatory disturbances (vascular pain, restless legs, or intermittent claudication); nor did we observe any trophic changes (skin lesions, ulcerations, gangrene) in patients with typical and uncomplicated rheumatoid arthritis.

The microscopical examination of the arterial biopsy seemed to explain the lack of clinical signs. The arteriosclerosis proved to be of the so-called Mönckeberg type, which occurs more frequently in the extremities and is characterized by degenerative necrotic changes in the media of the arteries followed by deposition of calcium in the necrotic areas. In contrast, lesions of the intima are relatively rare, although they cannot be entirely excluded. In the present study, for example, rather pronounced intimal thickening was observed.
The question still remains why this type of arteriosclerosis develops three times as frequently in steroidtreated patients with rheumatoid arthritis as in patients with the same disease but not treated with corticosteroids.

Are the degenerative processes with subsequent necrosis of the media of the arteries provoked and accelerated by the steroid therapy-or has the number of available calcium ions increased as a result of a general steroid-induced osteoporosis?

Both possibilities are open for discussion, even though the former, being the primary condition of arteriosclerosis, seems the more likely.

Two remarkable reports of animal experiments (Grossfeld, 1959; Wexler and Miller, 1958) support the present conclusions regarding the apparently harmful effect of cortcosteroids upon the arterial tissues.

\section{Summary}

A new largely asymptomatic side-effect of long-term treatment with corticosteroids is described.

By employing a soft exposure, radiographic evidence of arteriosclerosis in the region of the ankle joint was demonstrated three times as frequently in patients with rheumatoid arthritis who had been treated with steroids as in a control series. The arteriosclerosis was more extensive as well as more frequent in the patients treated with steroids.

An arterial biopsy demonstrated that a Mönckeberg's type of arteriosclerosis was involved; this is characterized by degenerative changes in the media, followed by calcium deposition. The arterial changes were apparently asymptomatic particularly as regards occlusive phenomena, although some intimal changes were observed.

\section{ADDENDUM}

Since this paper was submitted for publication we have seen two cases of typical gangrene of the toes in male patients with rheumatoid arthritis who were receiving long-term treatment with corticosteroids and who developed arteriosclerosis as described above.

In one patient (aged 59 years) amputation above the knee was necessary. The other patient (aged 61 years) had only the gangrenous first toe removed.

\section{References}

Adlersberg, D., Schaefer, L., ANd Drachman, S. R. (1950) J. Amer. med. Ass., 144 ,909 (Developmentment of hypercholestermia during cortisone and ACTH therapy)

GardNER, D. L. (1965) 'Pathology of the Connective Tissue Diseases'. Arnold, London

Grossfeld, H. (1959) Nature (Lond.), 184, 38 (Mechanism of the atherogenic action of excess hydrocortisone)

Skrifvars, B., Laine, V., AND Wegelius, O. (1969) Acta med. scand. 186, 145 (Sclerosis of the arteries of the extremities in rheumatoid arthritis)

WeXler, B. C., AND Miller, B. F. (1958) Science, 127, 590 (Severe arteriosclerosis and other diseases in the rat produced by corticotrophin) 